\title{
Bi-First: A simple and efficient algorithm to identify dissipated energy in impact problems
}

\author{
Z.-Q. Feng ${ }^{1}$, B. Magnain ${ }^{1}$, J.-M. Cros $^{1}$ and P. Joli ${ }^{2}$ \\ 1 Université d'Évry-Val d'Essonne, Laboratoire de Mécanique d'Évry, 40 rue du Pelvoux, 91020 Évry, France \\ 2 Université d'Évry-Val d'Essonne, Laboratoire IBISC, 40 rue du Pelvoux, 91020 Évry, France
}

Received 29 May 2007; accepted 1st October 2007

\begin{abstract}
The bi-potential method has been successfully applied for the modelling of frictional contact problems in static cases. This paper presents the extension of this method for dynamic analysis of impact problems with multiple deformable bodies. Instead of second order algorithms, a first order algorithm is applied for the numerical integration of the time-discretized equation of motion. The solution algorithm, named Bi-First, is simple and efficient. The principle of energy conservation for the given exemples is well preserved using the algorithm without any regularization. The numerical results also show clearly the physical energy dissipation introduced by frictional effects between the solids in contact.
\end{abstract}

Key words: Impact; energy dissipation; bi-potential method; time-integration

\section{Introduction}

Problems involving contact and friction are among the most difficult ones in mechanics and at the same time of crucial practical importance in many engineering branches. The main mathematical difficulty lies in the severe contact non-linearities because the natural first order constitutive laws of contact and friction phenomena are expressed by non-smooth multivalued force-displacement or force-velocity relations. In the last decade, substantial progress has been made in the analysis of contact problems using finite element procedures. A large number of algorithms for the numerical solution of the related finite element equations and inequalities have been presented in the literature. Review papers may be consulted for an extensive list of references [1-3]. See also the monographs by Kikuchi and Oden [4], Zhong [5] and Wriggers [6]. The popular penalty approximation and 'mixed' or 'trial-and-error' methods [7,8] appear, at first glance, suitable for many applications. But in this kind of method, the contact boundary conditions and friction laws are not satisfied accurately and it is tricky for the users to choose appropriate penalty factors. They may fail for stiff problems because of unpleasant numerical oscillations between contact statuses. The augmented Lagrangian method first appeared to deal with constrained minimization problems. Since friction problems are not minimization problems, the formulation needs to be extended. Alart and Curnier [9], Simo and Laursen [10] and De Saxcé and Feng [11] have obtained some extensions in mutually independent works. The first two works consist of applying Newton's method to the saddle-point equations of the augmented Lagrangian. De Saxcé and Feng proposed a theory called ISM (Implicit Standard Materials) and a bi-potential method, in which another augmented Lagrangian formulation was developed, which is essentially different from that of the first two works. In particular, in the bi-potential method, the frictional contact problem is treated in a reduced system by means of a reliable and efficient predictor-corrector solution algorithm. For the unilateral contact problems with friction, the classic approach is based on two minimum principles or two variational inequalities: the first for unilateral contact and the second for friction. The bi-potential method leads to a single displacement variational principle and a unique inequality. In consequence, the unilateral contact and the friction are coupled via a contact bi-potential. The application of the augmented Lagrangian method to the contact laws leads to an equation of projection onto Coulomb's cone, strictly equivalent to the original inequality [12]. For additional comments, see also the interesting discussion by Klarbring et al. $[13,14]$.

For dynamic implicit analysis in structural mechanics, the most commonly used time integration algorithm is the second order algorithm such as Newmark, Wilson, HHT. Wriggers et al. [15] have developed a radial return mapping scheme to deal with impact-contact problems. Laursen et al. [16-18] have considered dynamic impact under the auspices of a conservative system and have proposed the means to address the dynamic contact conditions so that they preserve the global conservation properties. The integration scheme is based on the second order algorithm. Some first order algorithms have also been proposed by Zienkiewicz et al. [19] and Jean [20] for time stepping in structural dynamics. 


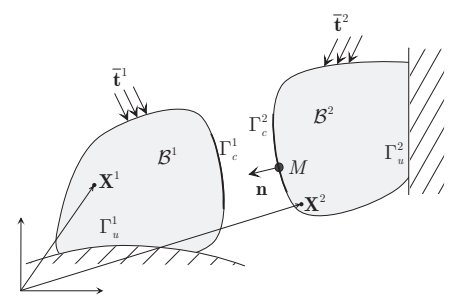

Fig. 1. Contact kinematics.

The aim of the present paper is to apply the bipotential method for contact modeling in dynamic cases in the field of Non-Smooth Dynamics using the first order algorithm for integration of the equation of motion. The algorithm developed is named Bi-First and is implemented into the finite element code FER/Contact, using $\mathrm{C}++$ with object oriented programming techniques. Two numerical examples are performed in this study to show the validity of the model developed. The first example concerns the oblique impact of an elastic plate onto a rigid surface with rebounding. The second example simulates the impact of two elastic cylinders in rigid walls. In order to show the physical energy dissipation by frictional effects and the behavior of the energy dissipation with respect to the friction coefficient, frictionless and frictional contact are considered for both examples.

\section{Problem setting}

\subsection{Contact kinematics}

In the following, basic definitions and notations used are described. Two deformable bodies $\mathcal{B}^{\alpha}$ (Fig. 1), $\alpha=1,2$, are considered. Each of them occupies the open, simply connected, bounded domain $\Omega^{\alpha} \subset \mathbb{R}^{3}$, whose generic point is denoted $\mathbf{X}^{\alpha}$. Furthermore, the solids are elastic and undergo large displacements. The boundary $\Gamma^{\alpha}$ of each body is assumed to be sufficiently smooth everywhere such that an outward unit normal vector, denoted by $\mathbf{n}^{\alpha}$, can be defined at any point $M$ on $\Gamma^{\alpha}$. At each time $t \in \mathbf{I}$, where $\mathbf{I}=[0, T]$ denotes the time interval corresponding to the loading process, the boundary $\Gamma^{\alpha}$ of the body $\mathcal{B}^{\alpha}$ can, in general, be split into three parts: $\Gamma_{u}^{\alpha}$ with prescribed displacements $\overline{\mathbf{u}}^{\alpha}, \Gamma_{t}^{\alpha}$ with prescribed boundary loads $\overline{\mathbf{t}}^{\alpha}$, and the potential contact surfaces $\Gamma_{c}^{\alpha}$ where the two bodies $\mathcal{B}^{1}$ and $\mathcal{B}^{2}$ may possibly come into contact at some time $t$ :

$$
\Gamma^{\alpha}=\Gamma_{u}^{\alpha} \cup \Gamma_{t}^{\alpha} \cup \Gamma_{c}^{\alpha} .
$$

The successive deformed configurations of $\mathcal{B}^{\alpha}$ are described at each time $t$ by the displacement fields $\mathbf{u}^{\alpha}$ defined on $\bar{\Omega}^{\alpha}$ (i.e. the closure of $\Omega^{\alpha}$ ). On the contact surface, a unique normal $\mathbf{n}$ directed towards $\mathcal{B}^{1}\left(\mathbf{n} \equiv \mathbf{n}^{2}\right)$ is defined and the tangential plane, orthogonal to $\mathbf{n}$ in $\mathbb{R}^{3}$, is denoted by $\mathbf{T}$. To construct an orthonormal local basis, two unit vectors $\mathbf{t}_{x}$ and $\mathbf{t}_{y}$ are defined within the plane T. For describing the frictional contact interactions that may occur on $\Gamma_{c}$, we introduce the relative velocity with respect to $\mathcal{B}^{2}$

$$
\dot{\mathbf{u}}=\dot{\mathbf{u}}^{1}-\dot{\mathbf{u}}^{2}
$$

where $\dot{\mathbf{u}}^{1}$ and $\dot{\mathbf{u}}^{2}$ are the instantaneous velocities of $\mathcal{B}^{1}$ and $\mathcal{B}^{2}$, respectively. Let $\mathbf{r}$ be the contact force distribution exerted on $\mathcal{B}^{1}$ at $M$ from $\mathcal{B}^{2}$. According to the actionreaction principle, $\mathcal{B}^{2}$ is subjected to the stress vector $-\mathbf{r}$. In the local coordinate system defined by the tangential plane $\mathbf{T}$ and the normal $\mathbf{n}$, any element $\dot{\mathbf{u}}$ and $\mathbf{r}$ may be uniquely decomposed as

$$
\begin{aligned}
& \dot{\mathbf{u}}=\dot{\mathbf{u}}_{t}+\dot{u}_{n} \mathbf{n}, \quad \dot{\mathbf{u}}_{t} \in \mathbf{T}, \quad \dot{u}_{n} \in \mathbb{R} \text {. } \\
& \mathbf{r}=\mathbf{r}_{t}+r_{n} \mathbf{n}, \quad \mathbf{r}_{t} \in \mathbf{T}, \quad r_{n} \in \mathbb{R} \text {. }
\end{aligned}
$$

\subsection{Contact law and friction rule}

The unilateral contact law is characterized by a geometric condition of non-penetration, a static condition of no-adhesion and a mechanical complementary condition. These three conditions are known as the Signorini conditions. The non-penetration condition constraints the displacement fields $\mathbf{u}^{\alpha}$ and is given by

$$
g(\mathbf{X})=\left(\mathbf{X}^{1}-\mathbf{X}^{2}\right) \cdot \mathbf{n} \geq 0
$$

where

$$
\mathbf{X}^{\alpha}(t)=\mathbf{X}^{\alpha}(t=0)+\mathbf{u}^{\alpha}
$$

The position vector $\mathbf{X}^{2}$ is found as the closest-point projection of the point $\mathbf{X}^{1} \in \Gamma_{c}^{1}$ on the surface $\Gamma_{c}^{2}$. Denoting by $h$ the initial gap obtained at the beginning of each time step.

$$
h=\left(\mathbf{X}^{1}-\mathbf{X}^{2}\right) \cdot \mathbf{n} \geq 0 .
$$

The impenetrability Signorini conditions are given by

$$
u_{n}+h \geq 0, \quad r_{n} \geq 0, \quad\left(u_{n}+h\right) r_{n}=0 .
$$

These conditions have to be satisfied at each time-instant $t \in \mathbf{I}$. Assume now that the bodies are initially in contact on a certain portion of $\Gamma_{c}$. On this part of $\Gamma_{c}$, the Signorini conditions turn into

$$
u_{n} \geq 0, \quad r_{n} \geq 0, \quad u_{n} r_{n}=0 .
$$

In general, at any time $t \in \mathbf{I}$, the potential contact surfaces $\Gamma_{c}^{\alpha}$ can be split into two disjoint parts: ${ }^{+} \Gamma_{c}$ where the bodies are already in contact and ${ }^{-} \Gamma_{c}^{\alpha}$ where the bodies are not in contact:

$$
\Gamma_{c}^{\alpha}={ }^{+} \Gamma_{c} \cup^{-} \Gamma_{c}^{\alpha} .
$$

In contrast to $\Gamma_{c}^{\alpha},{ }^{+} \Gamma_{c}$ and ${ }^{-} \Gamma_{c}^{\alpha}$ change in time $t$ and can be empty at some $t \in \mathbf{I}$. We must stress that with the formulation (9) only a loss of contact is allowed and the extension of the contact area cannot be modelled with these relations. In the case of dynamic analysis such as impact problems, the Signorini conditions can be formulated, on ${ }^{+} \Gamma_{c}$, in terms of relative velocity

$$
\dot{u}_{n} \geq 0, \quad r_{n} \geq 0, \quad \dot{u}_{n} r_{n}=0 \quad \text { on }{ }^{+} \Gamma_{c} .
$$




$$
\begin{aligned}
& \text { if } \quad r_{n}=0 \text { then } \dot{u}_{n} \geq 0 \\
& \begin{array}{ll}
\text { if } \quad r_{n}=0 \text { then } \dot{u}_{n} \geq 0 & \text { ! separating } \\
\text { elseif } \mathbf{r} \in \operatorname{int} K_{\mu} \text { then } \dot{u}_{n}=0 \text { and }-\dot{\mathbf{u}}_{t}=\mathbf{0} & \text { ! sticking }
\end{array} \\
& \text { else }\left(\mathbf{r} \in \text { bd } K_{\mu} \text { and } r_{n}>0\right) \\
& \left\{\dot{u}_{n} \geq 0 \text { and } \exists \dot{\lambda}>0 \text { such that }-\dot{\mathbf{u}}_{t}=\dot{\lambda} \frac{\mathbf{r}_{t}}{\left\|\mathbf{r}_{t}\right\|}\right\} \text { ! sliding } \\
& \text { endif }
\end{aligned}
$$

When $\dot{u}_{n} \geq 0$, the bodies are separating while they remain in contact for $\dot{u}_{n}=0$. The previous formulation of the Signorini conditions (11) can be combined with the sliding rule to derive the complete frictional contact law applicable on the contacting part of $\Gamma_{c}$. This complete law specifies possible velocities of bodies that satisfy impenetrability, non-adhesion and the sliding rule. Obviously, for a strictly positive gap $\left(u_{n} \geq 0\right)$, the normal relative velocity is arbitrary $\left(\dot{u}_{n} \in \mathbb{R}\right)$ and the normal reaction force is equal to zero $\left(r_{n}=0\right)$. Motions of bodies that are not in contact are arbitrary until contact is made. This choice is motivated by the fact that the emphasis is put on the definition of admissible evolutions for contacting bodies where the time-integration has to be performed. In the rest of the paper, a "minus" sign will always precede the relative tangential velocity $-\dot{\mathbf{u}}_{t}$ to emphasize its opposite direction to the friction force.

Classically, a rate independent dry friction law is characterized by a kinematic slip rule. In this work, the classical Coulomb friction rule is used. The set of admissible forces, denoted by $K_{\mu}$, is defined by

$$
K_{\mu}=\left\{\mathbf{r} \in \mathbb{R}^{3} \text { such that }\left\|\mathbf{r}_{t}\right\|-\mu r_{n} \leq 0\right\}
$$

$K_{\mu}$ is the so-called Coulomb's cone and is convex.

\subsection{Complete frictional contact law}

We consider now the previous friction law embedding an impenetrability condition for completeness. On the contact surface $\Gamma_{c}$, the sliding rule can be combined with the rate form of the Signorini conditions to obtain the frictional contact law that specifies possible scenarios on the contact area (stick, slip, separation). The multivalued nature of this strongly non-linear law makes problems involving frictional contact among the most difficult ones in solid mechanics. Two overlapped "if...then...else" statements can be used to write it analytically:

$$
\text { see equation (13) above }
$$

where "int $K_{\mu}$ " and "bd $K_{\mu}$ " denote the interior and the boundary of $K_{\mu}$, respectively. The multivalued character of the law lies in the first and the second part of the statement. If $r_{n}$ is null then $\dot{\mathbf{u}}$ is arbitrary but its normal component $\dot{u}_{n}$ should be positive. In other words, one single element of $\mathbb{R}^{3}(\mathbf{r}=\mathbf{0})$ is associated with an infinite number of velocity vectors $\dot{\mathbf{u}} \in \mathbb{R}^{3}$. The same arguments can be developed for the second part of the statement. The inverse law, i.e. the relationship $\mathbf{r}(-\dot{\mathbf{u}})$, can be written as:

$$
\begin{aligned}
& \text { if } \quad \dot{u}_{n}>0 \text { then } r_{n}=0 \\
& \text { elseif } \dot{\mathbf{u}}=\mathbf{0} \text { then } \mathbf{r} \in K_{\mu} \\
& \text { else }(\dot{\mathbf{u}} \in \mathbf{T}-\{\mathbf{0}\}) \\
& \left\{\dot{u}_{n} \geq 0 \text { and } \mathbf{r}_{t}=\mu r_{n} \frac{-\dot{\mathbf{u}}_{t}}{\left\|-\dot{\mathbf{u}}_{t}\right\|}\right\} \text { ! sliding } \\
& \text { endif } \\
& \text { ! separating } \\
& \text { ! sticking }
\end{aligned}
$$

The complete form of the frictional contact law involves three possible states, which are separating, contact with sticking, and contact with sliding. Only the last state produces energy dissipation.

\section{The bi-potential method}

De Saxcé and Feng [12] have shown that the contact law (13) is equivalent to the following differential inclusion:

$$
-\left(\dot{\mathbf{u}}_{t}+\left(\dot{u}_{n}+\mu\left\|-\dot{\mathbf{u}}_{t}\right\|\right) \mathbf{n}\right) \in \partial \bigcup_{K_{\mu}} \mathbf{r}
$$

where $\bigcup_{K_{\mu}} \mathbf{r}$ denotes the so-called indicatory function of the closed convex set $K_{\mu}$ :

$$
\bigcup_{K_{\mu}}(\mathbf{r})= \begin{cases}0 & \text { if } \mathbf{r} \in K_{\mu} \\ +\infty & \text { otherwise }\end{cases}
$$

The following contact bi-potential is obtained:

$$
b_{c}(-\dot{\mathbf{u}}, \mathbf{r})=\bigcup_{\mathbb{R}_{-}}\left(-\dot{u}_{n}\right)+\bigcup_{K_{\mu}}(\mathbf{r})+\mu r_{n}\left\|-\dot{\mathbf{u}}_{t}\right\|
$$

where $\left.\left.\mathbb{R}_{-}=\right]-\infty, 0\right]$ is the set of the negative and null real numbers.

In order to avoid nondifferentiable potentials that occur in nonlinear mechanics, such as in contact problems, it is convenient to use the Augmented Lagrangian Method [9-13]. For the contact bi-potential $b_{c}$, given by (17), provided that $\dot{u}_{n} \geq 0$ and $\mathbf{r} \in K_{\mu}$, we have:

$\forall \mathbf{r}^{\prime} \in K_{\mu}, \quad \varrho \mu\left(r_{n}^{\prime}-r_{n}\right)\left\|-\dot{\mathbf{u}}_{t}\right\|+\left(\mathbf{r}^{\prime}-(\mathbf{r}-\varrho \dot{\mathbf{u}})\right) \cdot\left(\mathbf{r}^{\prime}-\mathbf{r}\right) \geq 0$

where $\varrho$ is a solution parameter which is not user-defined. In order to ensure numerical convergence, $\varrho$ can be chosen as the maximum value of the diagonal terms of the local contact stiffness matrix. Taking account of the decomposition $(3,4)$, the following inequality has to be satisfied:

$$
\mathbf{r}^{\prime} \in K_{\mu}, \quad(\mathbf{r}-\tau) \cdot\left(\mathbf{r}^{\prime}-\mathbf{r}\right) \geq 0
$$




$$
\begin{array}{lll}
\text { if } & \mu\left|\boldsymbol{\tau}_{t}{ }^{i+1}\right|<-\tau_{n}^{i+1} \text { then } \mathbf{r}^{i+1}=0 & \text { ! separating } \\
\text { elseif } & \left|\boldsymbol{\tau}_{t}{ }^{i+1}\right|<\mu \tau_{n}^{i+1} \text { then } \mathbf{r}^{i+1}=\boldsymbol{\tau}^{i+1} & \text { ! sticking } \\
\text { else } & \mathbf{r}^{i+1}=\boldsymbol{\tau}^{i+1}-\frac{\left(\left\|\boldsymbol{\tau}_{t}^{i+1}\right\|-\mu \tau_{n}^{i+1}\right)}{\left(1+\mu^{2}\right)}\left(\frac{\boldsymbol{\tau}_{t}^{i+1}}{\left\|\boldsymbol{\tau}_{t}^{i+1}\right\|}+\mu \mathbf{n}\right) & \text { ! sliding }
\end{array}
$$

where the modified augmented surface traction $\tau$ is defined by

$$
\boldsymbol{\tau}=\mathbf{r}-\varrho\left(\dot{\mathbf{u}}_{t}+\left(\dot{u}_{n}+\mu\left\|-\dot{\mathbf{u}}_{t}\right\|\right) \mathbf{n}\right) .
$$

The inequality (19) means that $\mathbf{r}$ is the projection of $\boldsymbol{\tau}$ onto the closed convex Coulomb's cone:

$$
\mathbf{r}=\operatorname{proj}\left(\boldsymbol{\tau}, K_{\mu}\right) .
$$

For the numerical solution of the implicit equation (21), Uzawa's algorithm can be used, which leads to an iterative process involving one predictor-corrector step:

$$
\begin{aligned}
& \text { Predictor } \boldsymbol{\tau}^{i+1}=\mathbf{r}^{i}-\varrho^{i}\left(\dot{\mathbf{u}}_{t}^{i}+\left(\dot{u}_{n}^{i}+\mu\left\|-\dot{\mathbf{u}}_{t}^{i}\right\|\right) \mathbf{n}\right), \\
& \text { Corrector } \mathbf{r}^{i+1}=\operatorname{proj}\left(\boldsymbol{\tau}^{i+1}, K_{\mu}\right) .
\end{aligned}
$$

It is worth noting that, in this algorithm, the unilateral contact and the friction are coupled via the bi-potential. Another gist of the bi-potential method is that the corrector can be analytically found with respect to the three possible contact statuses: $\tau \subset K_{\mu}$ (contact with sticking), $\boldsymbol{\tau} \subset K_{\mu}^{*}$ (no contact) and $\boldsymbol{\tau} \subset \mathbb{R}^{3}-K_{\mu} \cup K_{\mu}^{*}$ (contact with sliding). $K_{\mu}^{*}$ is the polar cone of $K_{\mu}$. This corrector step is explicitly given as follows:

$$
\text { see equation (23) above }
$$

It is important to emphasize the fact that this explicit formula is valid for both $2 \mathrm{D}$ and $3 \mathrm{D}$ contact problems with Coulomb's friction and allows us to obtain very stable and accurate results.

\section{Finite element formulation of nonlinear structures}

\subsection{Total Lagrangian formulation}

In the linear analysis, a linear relation is assumed between strains and displacements. However, if there are large displacements and rotations, such as in the case of dynamic multibody contact problems, the nonlinear relation between strains and displacements cannot be ignored. Also, the equilibrium equation of internal and external forces should be considered in the deformed configuration. See the monographs by Crisfield [21] and Simo and Hughes [22] for more details on computational aspects of nonlinear problems. The geometrically nonlinear analysis may be described by using the total or the updated Lagrangian formulations. The total Lagrangian formulation is derived with respect to the initial configuration. The updated Lagrangian formulation is derived with respect to the current configuration. In other words, the total Lagrangian formulation constructs the tangent stiffness matrix with respect to the initial configuration. On the other hand, the updated Lagrangian formulation constructs the tangent stiffness matrix with respect to the current configuration. The updated Lagrangian formulation is computationally effective because it does not include the initial displacement matrix. In the total Lagrangian formulation, the initial configuration remains constant. This simplifies the computation. Therefore, the total Lagrangian formulation was selected in this work for the finite element discretization. In order to describe the geometrical transformation problems, the deformation gradient tensor is defined by

$$
\mathbf{\Phi}=\mathbf{I d}+\nabla \mathbf{u}
$$

where Id is the unity tensor and $\nabla \mathbf{u}$ the displacement gradient tensor. Because of large displacements and rotations, Green-Lagrangian strain is adopted for the nonlinear relationships between strains and displacements. We note $\mathbf{C}$ the stretch tensor or the right Cauchy-Green deformation tensor $\left(\mathbf{C}=\boldsymbol{\Phi}^{T} \boldsymbol{\Phi}\right)$. The Green-Lagrangian strain tensor $\mathbf{E}$ is defined by

$$
\mathbf{E}=\frac{1}{2}(\mathbf{C}-\mathbf{I})
$$

In the context of the finite element method and from equations $(24,25)$, the Green-Lagrangian strain includes formally linear and nonlinear terms in function of nodal displacements:

$$
\mathbf{E}=\left(\mathbf{B}_{L}+\frac{1}{2} \mathbf{B}_{N L}(\mathbf{u})\right) \mathbf{u}
$$

where $\mathbf{B}_{L}$ is the matrix which relates the linear strain term to the nodal displacements, and $\mathbf{B}_{N L}(\mathbf{u})$, the matrix which relates the nonlinear strain term to the nodal displacements. From equation (26), the incremental form of the strain-displacement relationship is

$$
\delta \mathbf{E}=\left(\mathbf{B}_{L}+\mathbf{B}_{N L}(\mathbf{u})\right) \delta \mathbf{u}
$$

In the case of elastic or hyperelastic laws, there exists an elastic potential function $W$ (or strain energy density function) which is a scalar function of the strain tensors, whose derivative with respect to a strain component determines the corresponding stress component. This can be expressed by

$$
\mathbf{S}=\frac{\partial W}{\partial \mathbf{E}}=2 \frac{\partial W}{\partial \mathbf{C}}
$$

where $\mathbf{S}$ is the second Piola-Kirchhoff stress tensor. In the particular case of isotropic Saint-Venant-Kirchhoff material models, we have

$$
W=\frac{1}{2} \mathbf{E}: \mathbf{D E} .
$$


So $\mathbf{S}$ can be written by

$$
\mathrm{S}=\mathrm{DE}
$$

where $\mathbf{D}$ denotes the usual material secant tangent. Using the principle of virtual displacement, the virtual work $\delta U$ is given as

$\delta U=\mathbf{M} \ddot{\mathbf{u}} \delta \mathbf{u}+\mathbf{A} \dot{\mathbf{u}} \delta \mathbf{u}+\int_{V_{0}} \mathbf{S} \delta \mathbf{E} d V-\mathbf{F}_{e x t} \delta \mathbf{u}-\mathbf{R} \delta \mathbf{u}=0$

where $V_{0}$ is the volume of the initial configuration, $\mathbf{F}_{\text {ext }}$ the vector of external loads, $\mathbf{R}$ the contact reaction vector, $\mathbf{M}$ the mass matrix, $\mathbf{A}$ the damping matrix, $\dot{\mathbf{u}}$ the velocity vector and $\ddot{\mathbf{u}}$ the acceleration vector. Substituting $\delta \mathbf{E}$ from equation (27) into equation (31) results in

$\delta U=\mathbf{M} \ddot{\mathbf{u}} \delta \mathbf{u}+\mathbf{A} \dot{\mathbf{u}} \delta \mathbf{u}+$

$$
\int_{V_{0}} \mathbf{S}\left(\mathbf{B}_{L}+\mathbf{B}_{N L}(\mathbf{u})\right) \delta \mathbf{u} d V-\mathbf{F}_{e x t} \delta \mathbf{u}-\mathbf{R} \delta \mathbf{u}=0 .
$$

The vector of internal forces is defined by

$$
\mathbf{F}_{\text {int }}=\int_{V_{0}} \mathbf{S}\left(\mathbf{B}_{L}+\mathbf{B}_{N L}(\mathbf{u})\right) d V .
$$

Since $\delta \mathbf{u}$ is arbitrary, a set of nonlinear equations can be obtained as

$$
\mathbf{M u ̈}+\mathbf{A} \dot{\mathbf{u}}+\mathbf{F}_{\text {int }}-\mathbf{F}_{\text {ext }}-\mathbf{R}=0 .
$$

It is noted that the stiffness effect is taken into account by the internal forces vector $\mathbf{F}_{\text {int }}$. Equation (34) can be transformed into

$$
\mathbf{M} \ddot{\mathbf{u}}=\mathbf{F}+\mathbf{R}, \quad \text { where } \mathbf{F}=\mathbf{F}_{\text {ext }}-\mathbf{F}_{\text {int }}-\mathbf{A} \dot{\mathbf{u}}
$$

with the initial conditions at $t=0$

$$
\dot{\mathbf{u}}=\dot{\mathbf{u}}_{0} \text { and } \mathbf{u}=\mathbf{u}_{0} .
$$

Taking the derivative of $\mathbf{F}_{\text {int }}$ with respect to the nodal displacements $\mathbf{u}$ gives the tangent stiffness matrix as

$\mathbf{K}=\frac{\partial \mathbf{F}_{i n t}}{\partial \mathbf{u}}=\int_{V_{0}}\left(\frac{\partial \mathbf{S}}{\partial \mathbf{u}}\left(\mathbf{B}_{L}+\mathbf{B}_{N L}(\mathbf{u})\right)+\mathbf{S} \frac{\partial \mathbf{B}_{N L}(\mathbf{u})}{\partial \mathbf{u}}\right) d V$.

In addition, by using equations $(27,30)$, the tangent stiffness matrix is in fact the sum of the elastic stiffness matrix $\mathbf{K}_{e}$, the geometric stiffness (or initial stress stiffness) matrix $\mathbf{K}_{\sigma}$ and the initial displacement stiffness matrix $\mathbf{K}_{u}$ :

$$
\mathbf{K}=\mathbf{K}_{e}+\mathbf{K}_{\sigma}+\mathbf{K}_{u}
$$

where

$$
\begin{aligned}
& \mathbf{K}_{e}=\int_{V_{0}} \mathbf{B}_{L}^{T} \mathbf{D B}_{L} d V, \\
& \mathbf{K}_{\sigma}=\int_{V_{0}} \mathbf{S} \frac{\partial \mathbf{B}_{N L}}{\partial \mathbf{u}} d V,
\end{aligned}
$$

$$
\mathbf{K}_{u}=\int_{V_{0}}\left(\mathbf{B}_{L}^{T} \mathbf{D} \mathbf{B}_{N L}+\mathbf{B}_{N L}^{T} \mathbf{D B}_{L}+\mathbf{B}_{N L}^{T} \mathbf{D B}_{N L}\right) d V
$$

\subsection{First order integration algorithm}

We can now integrate equation (35) between consecutive time configuration $t$ and $t+\Delta t$. The most common method to do that is the Newmark method which is based on a second order algorithm. However, in impact problems, higher order approximation does not necessarily mean better accuracy, and may even be superfluous. At the moment of a sudden change of contact conditions (impact, release of contact), the velocity and acceleration are not continuous, and excessive regularity constraints may lead to serious errors. For this reason, Jean [20] has proposed a first order algorithm which is used in this work. This algorithm is based on the following approximations:

$$
\begin{gathered}
\int_{t}^{t+\Delta t} \mathbf{M} d \dot{\mathbf{u}}=\mathbf{M}\left(\dot{\mathbf{u}}^{t+\Delta t}-\dot{\mathbf{u}}^{t}\right) \\
\int_{t}^{t+\Delta t} \mathbf{F} d t=\Delta t\left((1-\xi) \mathbf{F}^{t}+\xi \mathbf{F}^{t+\Delta t}\right) \\
\int_{t}^{t+\Delta t} \mathbf{R} d t=\Delta t \mathbf{R}^{t+\Delta t} \\
\mathbf{u}^{t+\Delta t}-\mathbf{u}^{t}=\Delta t\left((1-\theta) \dot{\mathbf{u}}^{t}+\theta \dot{\mathbf{u}}^{t+\Delta t}\right)
\end{gathered}
$$

where $0 \leq \xi \leq 1 ; 0 \leq \theta \leq 1$. In the iterative solution procedure, all the values at time $t+\Delta t$ are replaced by the values of the current iteration $i+1$; for example, $\mathbf{F}^{t+\Delta t}=$ $\mathbf{F}^{i+1}$. A standard approximation of $\mathbf{F}^{i+1}$ gives

$$
\begin{aligned}
\mathbf{F}^{i+1} & =\mathbf{F}_{i n t}^{i}+\frac{\partial \mathbf{F}}{\partial \mathbf{u}}\left(\mathbf{u}^{i+1}-\mathbf{u}^{i}\right)+\frac{\partial \mathbf{F}}{\partial \dot{\mathbf{u}}}\left(\dot{\mathbf{u}}^{i+1}-\dot{\mathbf{u}}^{i}\right) \\
& =\mathbf{F}_{i n t}^{i}-\mathbf{K}^{i} \Delta \mathbf{u}-\mathbf{A}^{i} \Delta \dot{\mathbf{u}} .
\end{aligned}
$$

Finally, we obtain the recursive form of (19) in terms of displacements:

$$
\begin{aligned}
\overline{\mathbf{K}}^{i} \Delta \mathbf{u} & =\overline{\mathbf{F}}^{i}+\overline{\mathbf{F}}_{a c c}^{i}+\mathbf{R}^{i+1} \\
\mathbf{u}^{i+1} & =\mathbf{u}^{i}+\Delta \mathbf{u}
\end{aligned}
$$

where the so-called effective terms are given by

$$
\begin{gathered}
\overline{\mathbf{K}}^{i}=\xi \mathbf{K}^{i}+\frac{\xi}{\theta \Delta t} \mathbf{A}^{i}+\frac{1}{\theta \Delta t^{2}} \mathbf{M}^{i}, \\
\overline{\mathbf{F}}_{a c c}^{i}=-\frac{1}{\theta \Delta t^{2}} \mathbf{M}^{i}\left\{\mathbf{u}^{i}-\mathbf{u}^{t}-\Delta t \dot{\mathbf{u}}^{t}\right\}, \\
\overline{\mathbf{F}}^{i}=(1-\xi)\left(\mathbf{F}_{\text {int }}^{t}+\mathbf{F}_{\text {ext }}^{t}\right)+\xi\left(\mathbf{F}_{\text {int }}^{i}+\mathbf{F}_{\text {ext }}^{t+\Delta t}\right) .
\end{gathered}
$$

At the end of each time step, the velocity is updated by

$$
\dot{\mathbf{u}}^{t+\Delta t}=\left(1-\frac{1}{\theta}\right) \dot{\mathbf{u}}^{t}+\frac{1}{\theta \Delta t}\left(\mathbf{u}^{t+\Delta t}-\mathbf{u}^{t}\right) .
$$

By setting $\theta=\frac{1}{2}$, this scheme is then called the implicit trapezoidal rule and it is equivalent to the Tamma - Namburu method in which the acceleration need not be computed [23]. See [24] for the interesting comments on time stepping algorithms and on energy conservation.

It is noted that equation (47) is strongly non-linear, because of large rotations and large displacements of solid, 
for instance in multibody contact/impact problems. Besides, as mentioned above, the constitutive law of contact with friction is usually represented by inequalities and the contact potential is even nondifferentiable. Instead of solving this equation in consideration of all nonlinearities at the same time, Feng [25] has proposed a solution strategy which consists in separating the nonlinearities in order to overcome the complexity of calculation and to improve the numerical stability. As $\Delta \mathbf{u}$ and $\mathbf{R}$ are both unknown, equation (47) cannot be directly solved. First, the vector $\mathbf{R}$ is determined by the bi-potential method in a reduced system, which only concerns contact nodes. Then, the vector $\Delta \mathbf{u}$ can be computed in the whole structure, using contact reactions as external loading. It is very important to note that, as opposed to the penalty method or Lagrange multiplier method, the bi-potential method neither changes the global stiffness matrix, nor increases the degrees of freedom. One consequence of this interesting property is that it is easy to implement contact and friction problems in an existing general-purpose finite element code by this method. In addition, the solution procedure is more stable because of the separation of nonlinearities and improved numerical algorithms for calculation of contact reactions.

\subsection{Energy computation}

After determining the displacement and the velocity fields, we can calculate different energies. The total elastic strain energy of the contact bodies (discretized by $n_{e l}$ finite elements) is then written by

$$
E_{e}=\sum_{e=1}^{n_{e l}} \int_{\Omega_{e}} W_{e} d \Omega .
$$

The total kinetic energy can be calculated at the global level by

$$
E_{k}=\frac{1}{2} \dot{\mathbf{u}}^{T} \mathbf{M} \dot{\mathbf{u}} .
$$

Finally, the total energy of the system of solids is

$$
E_{t}=E_{e}+E_{k}
$$

The case of interest for the analysis presented below corresponds to the homogeneous Neumann problem, characterized by no imposed boundary displacements and no external loading. In addition, if frictionless contact is considered, the total energy should be conserved. For the given examples, this fundamental energy conservation property has been observed.

\section{Numerical results}

The algorithms presented above have been implemented and tested in the finite element code FER/Contact [26]. Many application examples, in static or quasi-static cases, have been carried out using the present method $[25,27,28]$.

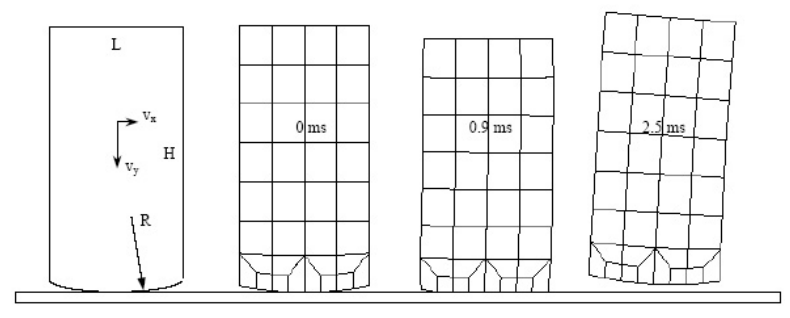

Fig. 2. Oblique impact of an elastic plate: geometry and deformed shapes vs time.

Table 1. Comparison of CPU time.

\begin{tabular}{|l|l|c|}
\hline Method & Computer & CPU time (s) \\
\hline Ko \& Kwak [29] & CRAY 2S/4-128 & 19000 \\
\hline Kim \& Kwak [30] & HP 720 & 430 \\
\hline present & PC Pentium $4 / 2.8 \mathrm{GHz}$ & 7 \\
\hline
\end{tabular}

To illustrate the behavior of a contact/impact simulation by the Bi-First algorithm described above, we consider two example applications. For both cases, we assume that no amortissement exists except for Coulomb friction between contact surfaces, i.e. $\mathbf{A}=0$ in equations $(34,35,48)$.

\subsection{Oblique impact of an elastic plate with rebounding}

The first example of dynamics analysis will be presented to show the validity and efficiency of the model developed. The problem concerns the oblique impact of an elastic plate onto a rigid surface with rebounding. This example has been proposed and studied by Kwak et al. [29,30] using Linear Complementarity Problem (LCP) formulation. The geometric configuration and successive deformed meshes are displayed in Figure 2. For comparison purposes, we have used the same mesh as in [30]. The characteristics of this example are: Young's modulus $E=10^{7} \mathrm{~Pa}$, Poisson's ratio $v=0.25$, mass density $\rho=1000 \mathrm{~kg} / \mathrm{m}^{3}$, Friction coefficient $\mu=0.1$, Initial velocity: $v_{x}=3 \mathrm{~m} / \mathrm{s}, v_{y}=-5 \mathrm{~m} / \mathrm{s}$. The geometric sizes are: $L=0.04 \mathrm{~m}, H=0.08 \mathrm{~m}$, radius $R=0.101 \mathrm{~m}$, thickness $e=0.01 \mathrm{~m}$. The total simulation time is $3.10^{-3} \mathrm{~s}$ and the solution parameters are: $\Delta t=10^{-5} \mathrm{~s}, \xi=\theta=0.5$. The plate is modeled by 54 nodes and 37 linear quadrilateral plane stress elements (Fig. 2). The performance of the present approach in terms of CPU time, as compared to Kwak's solutions, is reported in Table 1, which shows the efficiency of the proposed method. It is noted from Figure 3 that the total energy is dissipated by frictional effects and the dissipated energy is calculated quantitatively. It is also interesting to examine another question: is the dissipated energy monotone to the friction coefficient? The answer is no according to numerical results. The proof is illustrated by Figure 4 which shows the evolution of the dissipated energy with respect to the friction coefficient. In fact, when the friction coefficient increases, the friction forces increase. However, the tangential slips will decrease. We know that the dissipated energy de- 


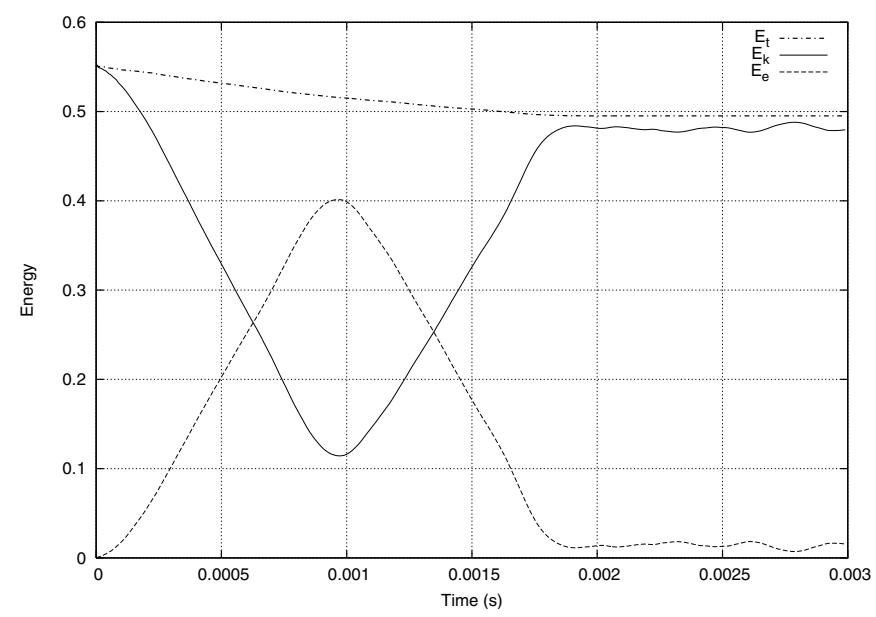

Fig. 3. Energy evolution vs time.

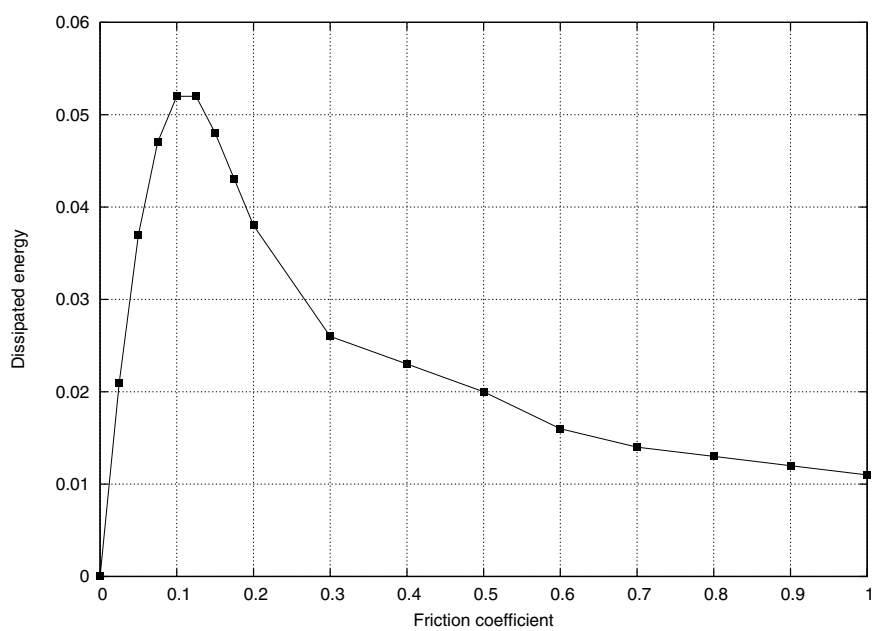

Fig. 4. Evolution of the dissipated energy with respect to the friction coefficient.

pends not only on the friction forces but also on the tangential slips on the contact nodes, so it is understandable to have the behavior as shown in Figure 4.

\subsection{Impact between two cylinders}

The second example simulates the impact of two cylinders inside rigid walls. In doing so, we wish to further explore the performance of the present method in a general situation with complicated contact sequences. The problem is displayed in Figure 5. Dimensionless data are intentionally used. The cylinders have a diameter of 2 . The SaintVenant-Kirchhoff material model is assumed for both cylinders with material constants: $E=2700, v=0.33$, and mass density $\rho=1$. The left cylinder is given an initial velocity of $v_{x}=1, v_{y}=-2$, hitting the bottom rigid wall and afterwards the right cylinder as depicted in Figure 5. The total simulation time is 15 and the solution parameters are: $\Delta t=10^{-3}, \xi=\theta=0.5$. Figure 6 shows the deformed configurations of the two cylinders A and $\mathrm{B}$ at different times $(1.558,2.898,5.278,6.298)$ cor-

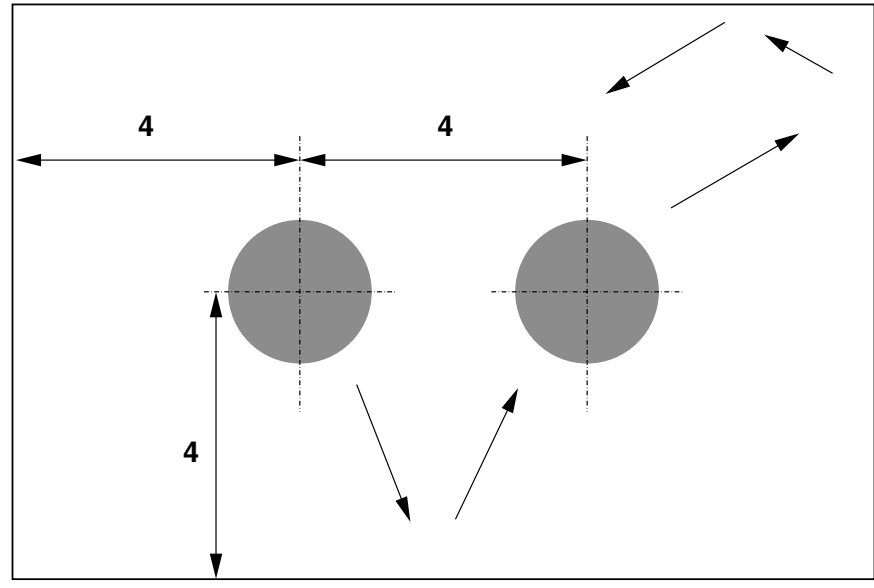

Fig. 5. Impact of two cylinders inside rigid walls.

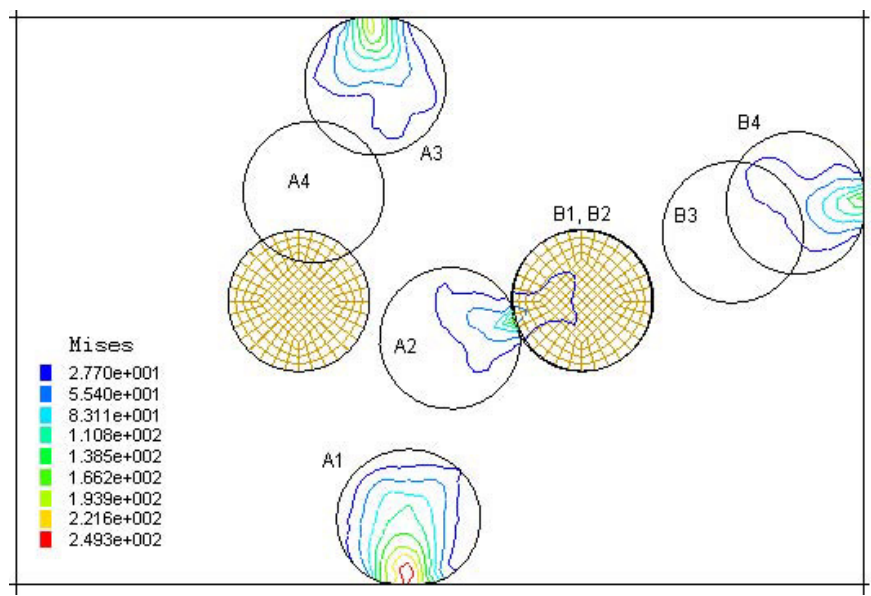

Fig. 6. Deformed configurations at different times (with friction).

responding respectively to $\left(A_{1}, B_{1}\right),\left(A_{2}, B_{2}\right),\left(A_{3}, B_{3}\right)$ and $\left(A_{4}, B_{4}\right)$ as shown in the figure. The isolines represent the distribution of the Mises stress. Figure 7 shows the plots of the kinetic energy $E_{k}$, the elastic strain energy $E_{e}$ and the total energy $E_{t}$. We can observe clearly that the total energy is perfectly conserved in the case of frictionless contact. However, in the case of frictional contact $(\mu=0.2)$, the total energy decreases at each shock (Fig. 8). So the energy is dissipated by frictional effects as expected.

It is interesting to note from Figure 7 that the left cylinder hits another one at $t=2.45$. On the other hand, $t=2.8$ in the case of frictional contact as indicated in Figure 8. This fact can be explained as follows: because of friction forces, the rebounding direction is changed such that the running distance of the left cylinder from the bottom wall to the right cylinder becomes longer. Thus, it takes more time to hit each other. Figure 9 shows the distribution of the shear stress of the left cylinder when it hits the bottom wall. Without friction, the distribution is symmetric, but this is not true with friction. Once again, the frictional effects are apparently demonstrated. 


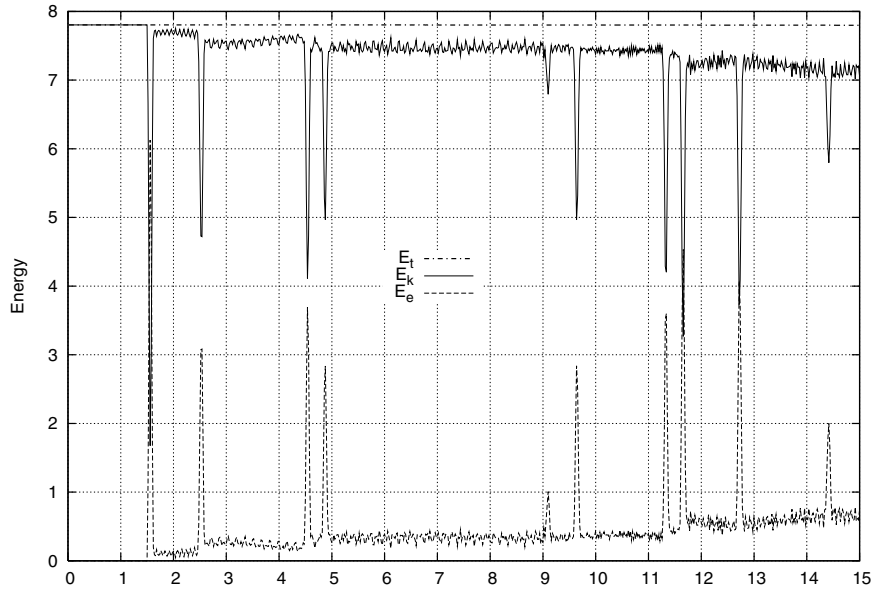

Fig. 7. Energy evolution without friction $(\mu=0.0)$.

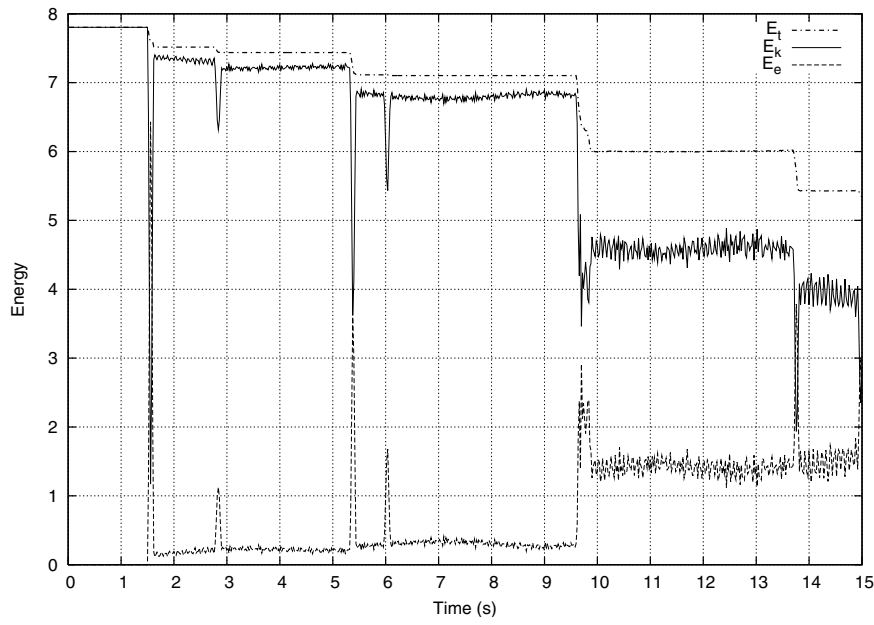

Fig. 8. Energy evolution with friction $(\mu=0.2)$.

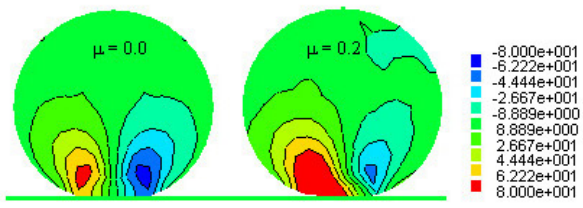

Fig. 9. Distribution of shear stress at $t=1.56$.

\section{Conclusion}

In this paper, we have presented the recent development of the bi-potential method applied to dynamic analysis of two-dimensional contact problems with Coulomb friction. The Bi-First algorithm has been described and investigated numerically for two problems using different coefficients of friction. From numerical experiments, we have found that:

- The total energy is well conserved in the case of frictionless contact of solids.

- The Bi-First algorithm permits to determine quantitatively the physical energy dissipation by frictional effects.
- The dissipated energy is not monotone with respect to the friction coefficient.

- The Bi-First algorithm is simple and efficient:

- no modification of the global stiffness matrix;

- no regularization of contact and friction laws;

- accurate calculation of contact forces in a reduced system;

- first order time stepping instead of second or higher order integration.

We have felt that this approach could easily be extended to three-dimensional dynamic contact problems including nonlinear material constitutive laws and more complex frictional models [31]. This work is being undertaken.

\section{References}

1. Z.H. Zhong, J. Macherle, Static contact problems - a review. Eng. Computation 9, 3-37 (1992)

2. A. Klarbring, Mathematical programming in contact problems, In MH. Aliabdali and CA. Brebbia, editors, Computational methods in contact mechanics, pages 233-263. Southampton: Computational Mechanics Publications (1993)

3. P. Wriggers, Finite element algorithms for contact problems. Arch. Comput. Method. E. 2, 1-49 (1995)

4. N. Kikuchi, J.T. Oden, Contact problems in elasticity: A study of variational inequalities and finite elements, Philadelphia: SIAM (1988)

5. Z.H. Zhong, Finite element procedures in contact-impact problems, Oxford University Press (1993)

6. P. Wriggers, Computational contact mechanics (John Wiley \& Sons, 2002)

7. A.B. Chaudhary, K.J. Bathe, A solution method for static and dynamic analysis of three dimensional contact problems with friction. Comput. Struct. 24, 855-873 (1986)

8. H.A. Parisch, Consistent tangent stiffness matrix for three-dimensional non-linear contact analysis. Int. J. Numer. Meth. Eng. 28, 1803-1812 (1989)

9. P. Alart, A. Curnier, A mixed formulation for frictional contact problems prone to Newton like solution methods. Comput. Method Appl. M. 92, 353-375 (1991)

10. J.C. Simo, T.A. Laursen, An augmented Lagrangian treatment of contact problems involving friction. Comput. Struct. 42, 97-116 (1992)

11. G. de Saxcé, Z.-Q. Feng, New inequality and functional for contact with friction: The implicit standard material approach. Mech. Struct. Mach. 19 301-325 (1991)

12. G. de Saxcé, Z.-Q. Feng, The bi-potential method: a constructive approach to design the complete contact law with friction and improved numerical algorithms. Mathematical and Computer Modeling 28, 225-245 (1998)

13. A. Klarbring, Mathematical programming and augmented Lagrangian methods for frictional contact problems. In A. Curnier, editor, Contact Mechanics Int. Symp. (1992) PPUR.

14. P.W. Chritensen, A. Klarbring, J.S. Pang, N.N. Strömberg, Formulation and comparison of algorithms for frictional contact problems. Int. J. Numer. Meth. Eng. 42, 145-173 (1998) 
15. P. Wriggers, T. Vu Van, E. Stein, Finite element formulation of large deformation impact contact problems with friction. Comput. Struct. 37, 319-331 (1990)

16. T.A. Laursen, V. Chawla, Design of energy conserving algorithms for frictionless dynamic contact problems. Int. J. Numer. Meth. Eng. 40, 863-886 (1997)

17. T.A. Laursen, G.R. Love, Improved implicit integrators for transient impact problems geometric admissibility within the conserving framework. Int. J. Numer. Meth. Eng. 53, 245-274 (2002)

18. G.R. Love, T.A. Laursen, Improved implicit integrators for transient impact problems: dynamic frictional dissipation within an admissible conserving framework. Comput. Method. Appl. M. 192, 2223-2248 (2003)

19. O.C. Zienkiewicz, W.L. Wood, L.W. Hine, R.L. Taylor, A unified set of single step algorithms. part 1. general formulation and application. Int. J. Numer. Meth. Eng. 20, 1529-1552 (1984)

20. M. Jean, Dynamics with partially elastic shocks and dry friction: double scale method and numerical approach, In 4th Meeting on unilateral problems in structural analysis (1989)

21. M.A. Crisfield, Non-linear finite element analysis of solid and structures (Wiley, 1991)

22. J.C. Simo, T.J.R. Hughes, Computational inelasticity, (Springer-Verlag, New York, 1998)

23. K.K. Tamma, R.R. Namburu, A robust self - starting explicit computational methodology for structural dynamic applications: architecture and representations. Int. J. Numer. Meth. Eng. 30, 1441-1454 (1990)
24. J.C. Simo, K.K. Wong, Unconditionally stable algorithms for rigid body dynamics that exactly preserve energy and momentum. Int. J. Numer. Meth. Eng. 31, 19-52 (1991)

25. Z.-Q. Feng, 2D or 3D frictional contact algorithms and applications in a large deformation context. Commun. Numer. Meth. En. 11, 409-416 (1995)

26. Z.-Q. Feng, http://gmfe16. cemif.univ-evry.fr:8080/ feng/ FerImpact.html

27. Z.-Q. Feng, Some test examples of $2 D$ and $3 D$ contact problems involving coulomb friction and large slip. Mathematical and Computer Modeling 28(4-8), 469477 (1998) Special issue: Recent Advances in Contact Mechanics.

28. Z.-Q. Feng, F. Peyraut, N. Labed, Solution of large deformation contact problems with friction between Blatz-Ko hyperelastic bodies. Int. J. Eng. Sci. 41, 2213-2225 (2003)

29. S.H. Ko, B. Kwak, Frictional dynamic contact analysis using finite element nodal displacement description. Comput. Struct. 42, 797-807 (1992)

30. J.O. Kim, B. Kwak, Dynamic analysis of two-dimensional frictional contact by linear complementarity problem formulation. Int. J. Solids Struct. 33, 4605-4624 (1996)

31. M. Hjiaj, Z.-Q. Feng, G. de Saxcé, Z. Mróz, Theredimensional finite element computations for frictional contact problems with non-associated sliding rule. Int. J. Numer. Meth. Eng. 60, 2045-2076 (2004) 